\title{
Intercavity Stimulated Scattering in Planar FEM as a Base for Two-Stage Generation of Submillimeter Radiation
}

\author{
Andrey Arzhannikov \\ Budker Institute of Nuclear Physics SB RAS, Novosibirsk State University \\ Russian Federation
}

\section{Introduction}

Far infrared and submillimeter radiation with wavelength from $30 \mu \mathrm{m}$ up to $300 \mu \mathrm{m}$ reveals possibilities for new technologies and registration methods inaccessible earlier. One can use this terahertz radiation (THR) to investigate properties of substances and materials such as semiconductors, paper, plastics, which are opaque in the visible range. The other important moment is that the eigenfrequencies of characteristic vibrations of complex molecules belong to the terahertz region. It means that application of THR opens up possibilities of purposive influence upon organic molecules including DNA and RNA. In medicine the terahertz radiation can be used for visualization of healthy and defective tissues, as well as an instrument of therapy and surgery.

There are various methods of generation of the terahertz radiation in the pointed wavelength band and a choice of one of them strongly depends on requirements of users for parameters of the radiation. From one hand, for the case of small generated power it can be done by solid structure lasers (Kohler et al., 2002) or by back wave oscillators (Dobroiu et al., 2004). From other hand, to generate the terahertz radiation of high level power one has to create very huge installations with multi-megavolt electron accelerators (Minehara et al., 2005) and (Vinokurov et al., 2006). As one of appropriate solving the problem of generation of the high power terahertz radiation we proposed (Arzhannikov et al., 2006) to use a twostage scheme of generation of short wavelength radiation by scattering an EM-wave on a beam of relativistic electrons for the case when at the first stage a high current sheet beam drives a free electron maser of planar geometry operated with two-dimensional distributed feedback at 4-mm wavelength (Arzhannikov et al., 1992, 1995, 2003). Theoretical analysis (Ginzburg et al., 1999) and experimental investigations (Arzhannikov et al., 2008) clear demonstrated that the free electron maser of planar geometry is truly appropriate oscillator for 4-mm radiation band. The key feature of our proposal on two-stage generation is to use two planar generators pumped by sheet beams with a few kAmps currents which plane resonators are combined as it was described for a multichannel generator of mm-wave radiation (Ginzburg et al., 2001) . 


\section{Proposed process and main experimental parameters}

\subsection{Wavelength bands of generated radiation}

To start our analysis of opportunity of the proposed two-stage scheme we need to outline the wavelength bands that can be covered by the two-stage generation at the experimental conditions of the ELMI-device. At the first stage of the two-stage process a free electron maser has to be used at the parameters of recent experiments at the ELMI-device to generate the radiation with the wavelength $\lambda_{0}=4 \mathrm{~mm}$ (Arzhannikov et al., 2008). If one assumes that this radiation will be scattered on the electrons with kinetic energy about of $1 \mathrm{MeV}$, one can estimate the output radiation wavelength at the second stage of generation.

For the double Doppler Effect the wavelength conversion is expressed by the following formula:

$$
\lambda=\lambda_{0}\left(1-\beta \cos \theta_{s}\right) /\left(1-\beta \cos \theta_{i}\right),
$$

where $\beta=\mathrm{v} / \mathrm{c}, \mathrm{v}$ - velocity of the beam electrons, $\mathrm{c}$ - velocity of light, is a $\beta$-factor of the beam electrons, $\theta_{\mathrm{i}}, \theta_{\mathrm{s}}$ - angles of incident and scattered radiation respectively counted off from the direction of the electron velocity vector (see Fig.1). For the special cases of backscattering and $90^{\circ}$-scattering the Doppler formula can be written as

$$
\lambda \approx \lambda_{0} /\left(4 \gamma^{2}\right) \text { and } \lambda \approx \lambda_{0} /\left(2 \gamma^{2}\right)
$$

respectively, where $\gamma=1 / \sqrt{1-\beta^{2}}$ is a E-beam relativistic factor. The expected wavelengths of the radiation at output of the second stage as the function of the $\gamma$-factor is presented in Fig.1.

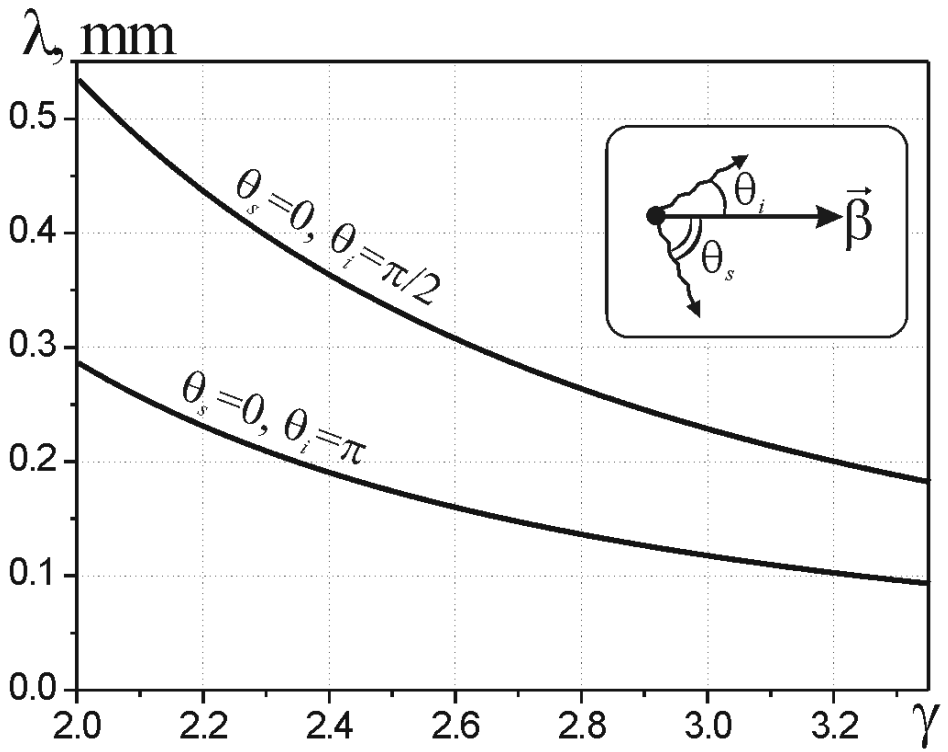

Fig. 1. Conversed wavelength due to scattering of 4-mm radiation by E-beam as the function of $\gamma$-factor of the beam electrons. 
It is clear that one can obtain radiation in the band of $0.1 \div 0.3 \mathrm{~mm}$ by scattering the incident radiation in the direction opposite to the beam electron velocity at various values of the electron relativistic factor. If the incident radiation is scattered in the transverse direction to the beam electron velocity the radiation wavelength should be shifted to the band of $\sim 0.2 \div 0.5 \mathrm{~mm}$.

\subsection{Schematic of the proposed experiments}

Schematic drawings of experimental realization of submm generation for these two wavelength bands are presented in Fig.2 and Fig.3, respectively. The Fig.2 illustrates the variant of two-stage generation for the band of $0.1 \div 0.3 \mathrm{~mm}$ using backscattering of $4-\mathrm{mm}$ radiation.

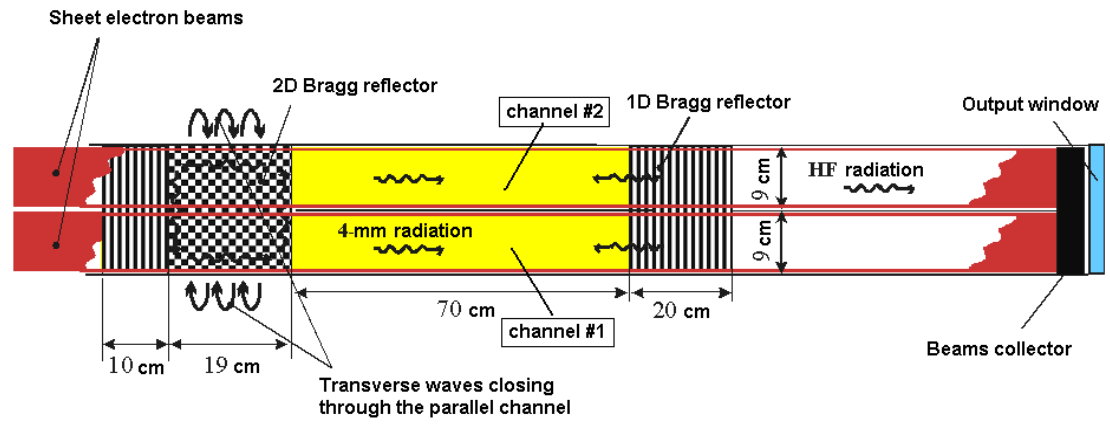

Fig. 2. Scheme of two-stage generation for the band of $0.1 \div 0.3 \mathrm{~mm}$.

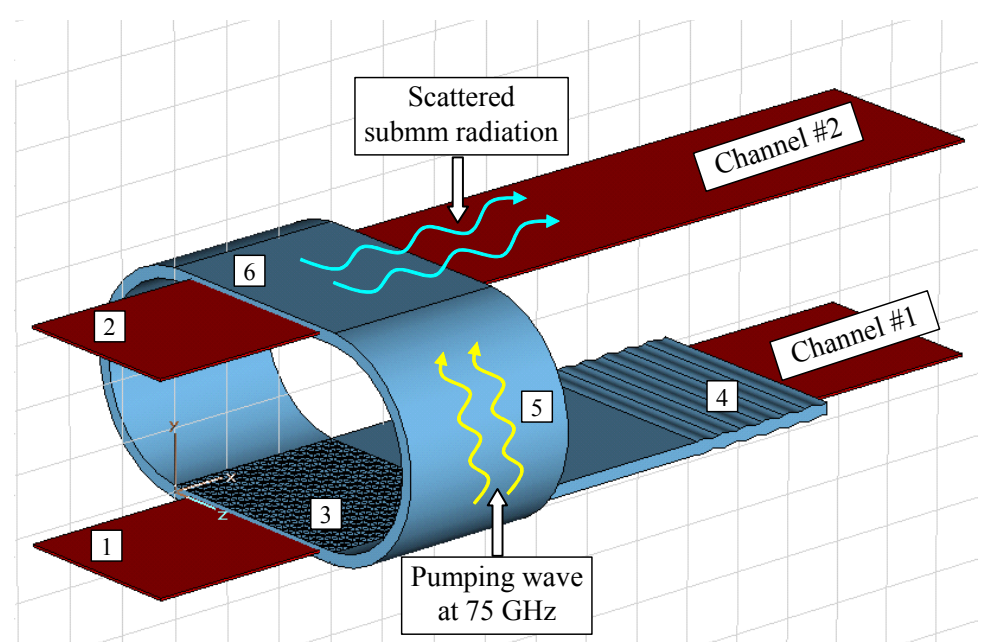

Fig. 3. Scheme of two-stage generation for the band of $0.2 \div 0.5 \mathrm{~mm}$ :

1) sheet REB for driving the planar FEM-generator; 2) sheet REB for mm-wave scattering;

3) 2-D Bragg reflector; 4) 1-D Bragg reflector; 5) feedback circuit; 6) place of scattering 
Fig. 3 presents the variant of generation for the band of $0.2 \div 0.5 \mathrm{~mm}$ where the radiation is scattered at the angle 90 .

For both variants we suppose to use sheet beams with $3 \div 4 \mathrm{~mm}$ thickness and $10 \div 20 \mathrm{~cm}$ width and a current density more than $1 \mathrm{kA} / \mathrm{cm}^{2}$. The E-beams pass the slit channels at presence of longitudinal guiding magnetic field with the strength greater than 1.0T. In the channel \#1 of both variants there is an undulator transverse component of the magnetic field that allows one to generate 4 -mm radiation with efficiency $10 \div 15 \%$. The energy density of 4 $\mathrm{mm}$ radiation inside the resonator of these FEM generators has a level which corresponds to the electric field strength $10^{5} \div 10^{6} \mathrm{~V} / \mathrm{cm}$ (Arzhannikov et al., 2003) and the same value of the strength must be in the channels \#2 of both variants.

In further analysis we shall concentrate our attention on using backscattering of 4-mm radiation that schematic is presented by Fig. 2. Main feature of the electrodynamics system for our two-stage experiments is to use Bragg reflectors in a resonator for 4-mm wave generation. Geometrical parameters of these 4-mm radiation reflectors constructed of the pair of Bragg gratings were chosen through computer simulations and their frequency selecting properties were measured on a special tested bench. Widths and lengths of the vacuum channels for passing the electron beams in were also chosen on the base of computer simulations and experimental tests.

\subsection{Computer simulations and experiments on simultaneous generation and transport of two sheet beams}

Before the investigations of two-stage generation by using the backscattering process, we have to design and to construct the accelerating diode suitable for simultaneous generation of two high-current sheet beams and to determine conditions for stable equilibrium transport of intense sheet electron REBs in the moderate magnetic fields inside the slit vacuum channels. Solving these two problems is described here.

\subsubsection{Computer simulations}

One of the key problems in generation of high power REBs suitable to produce THzradiation in frame of the two-stage scheme is to achieve limit brightness of the beam that is proportional to the current density of the beam $j$ and inversely proportional to the square of electron angular divergence $\theta^{2}$. Simple estimations have shown that the level of the beam density $j \sim 3 \mathrm{KA} / \mathrm{cm}^{2}$ at the spread of longitudinal velocities of the beam electrons $\Delta V_{\|} / V_{\|} \approx \frac{\theta^{2}}{2}<10^{-3}$ has to be achieved for acceptable efficiency of the wave energy transfer from the beam to the $\mathrm{THz}$ band radiation (Arzhannikov et al., 2006). It should be noted that to generate mm-wave radiation the value of this spread about $5 \cdot 10^{-2}$ is sufficient. Previous analytical consideration and computer simulations (Arzhannikov \& Sinitsky, 1996) showed that it was possible to reduce the angular divergence below the value $\theta \sim 2 \times 10^{-2}$ in case of the electron beam generated in the magnetically insulated diode with ribbon geometry at the diode voltage $1 \mathrm{MV}$ and relatively low electron current density $150 \mathrm{~A} / \mathrm{cm}^{2}$ in the magnetic field $0.6 \mathrm{~T}$ inside the slit channel. It was achieved by proper choice of the diode geometry and configuration of the magnetic field which set conditions for subtraction of contributions to the angular electron divergence from the electric and magnetic fields 
inhomogeneities. In the case of four beams generated simultaneously in a single uniform accelerating diode in the results of computer simulations we have demonstrated the possibility to reach sufficiently high brightness of the beams adequate for generating $\mathrm{mm}$ wave radiation. To investigate the prospects of such beams application for two-stage scheme of $\mathrm{THz}$ - wave generation we have performed computer modelling of simultaneous generation of two sheet beams in the magnetically insulated diode and the output of these beams in narrow slit channels. Obtained results confirmed the possibility to achieve the level of the angular divergence $\theta \sim 5 \cdot 10^{-2}\left(\Delta V_{\|} / V_{\|} \sim 10^{-3}\right)$ at a considerably high current density about $1 \mathrm{kA} / \mathrm{cm}^{2}$ in the magnetic field $1.7 \mathrm{~T}$ (Arzhannikov et al., 2007). Another important problem that has to be solved is the transport of the sheet beam in the slit channel at a stable equilibrium. It was a subject of theoretical and experimental investigations described in (Arzhannikov et al., 1990, 2007) and (Sinitsky et al., 2008). For our case we simulated the beam transport by solving 2-D Poisson equation for homogeneous current and space charge densities of the beam with sharp borders inside the rectangular liner with perfectly conducting walls. When self electric and magnetic fields are small in comparison with the external guiding magnetic field directed along the channel axis, the current and charge densities remain homogeneous along the beam pass but the beam border is deformed by the drift motion of the electrons and the displacement by self magnetic field of the beam :

$$
\overrightarrow{V_{\perp}}=c \frac{\vec{E} \times \vec{H}_{0}}{H_{0}^{2}}+v_{\|} \frac{\vec{H}}{H_{0}}=c \frac{\vec{E} \times \vec{H}_{0}}{H_{0}^{2}}\left(\frac{1}{\gamma^{2}}-f\right)
$$

where $\vec{E}$ and $\vec{H}$ - self beam fields, $\vec{H}_{0}$ - homogeneous external field, $f$-neutralization degree of the beam space charge, $\gamma$ - relativistic factor of the electrons. The Fig. 4 demonstrates the evolution of the cross section shape for the beam with the electron energy $0.8 \mathrm{MeV}$ beam current $3 \mathrm{kA}$ and initial cross section $0.4 \times 6.6 \mathrm{~cm}$ along the channel length for three distances $Z$ from the entrance of the channel and for three values of neutralization degree $f$.

$\mathrm{f}=1$
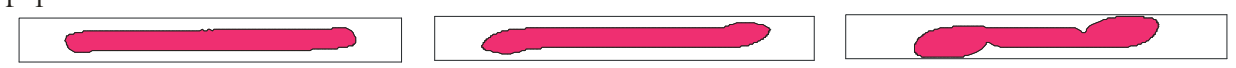

\section{$\mathrm{f}=0.5$}

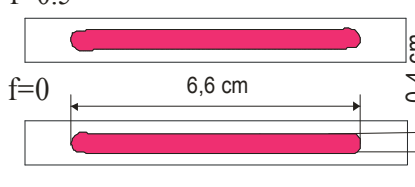

$$
\mathrm{Z}=17 \mathrm{~cm}
$$

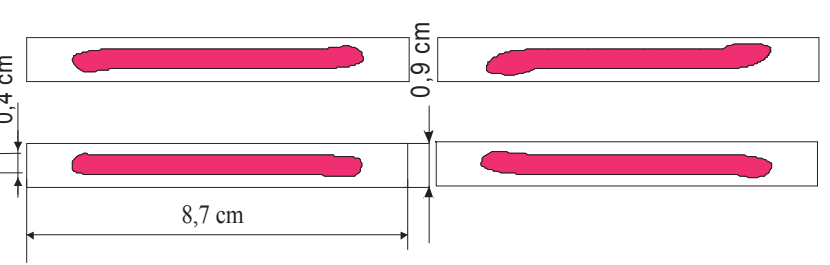

$\mathrm{Z}=50 \mathrm{~cm}$

$\mathrm{Z}=140 \mathrm{~cm}$

Fig. 4. Cross section shapes of the beam for three $Z$ coordinates along the channel at three values of the space charge neutralization $\mathrm{f}$. 
As it is seen the substantial shape deformations for $f=0$ and $f=0.5$ are expected only at the end of the channel $(\mathrm{Z}=140 \mathrm{~cm})$ while for $f=1$ they occur just at $\mathrm{Z}=50 \mathrm{~cm}$. It should be noted that to keep the beam shape unchangeable it is necessary to have beam thickness equal to $3 / 4$ of the channel gap. Unfortunately we can not satisfy this requirement because in the case of the FEM application the beam border should oscillate in the undulator field with the amplitude $\sim 0.1 \mathrm{~cm}$ and the electrons should have perpendicular Larmor radius $\sim 0.1 \mathrm{~cm}$ while the channel gap should not exceed 2-3 wavelength of the generated radiation $(4 \mathrm{~mm})$. Thus we have advisedly chosen nonequilibrium shape of the beam assuming its deformations on the length of the FEM resonator $(70 \mathrm{~cm})$ would be acceptable.

\subsubsection{Experiments on simultaneous generation and transport of two beams}

The experiments on the simultaneous generation of two sheet beams and their transport in slit vacuum channels were realized basing on the results of computer simulations. Schematic drawing of these experiments is presented in Fig. 5. (Arzhannikov et al., 2007 and Sinitsky et al., 2008). Two sheet beams are generated by two vertically elongated cathodes placed one over another (see side view). These cathodes are made of a fibrous graphite material to ensure homogeneous emission from their surfaces. The guiding magnetic field has adiabatic growth from $0.35 \mathrm{~T}$ in the diode up to $1.7 \mathrm{~T}$ in the channel that provides magnetic compression of the beam and rise of its current density up to $1-1.5 \mathrm{kA} / \mathrm{cm}^{2}$. According to simulations for such magnetic field growth the pitch angle of a main part of the beam electrons should not exceed a few degrees. The outer areas of the beam cross sections are cut off in special graphite formers at the beam entrances into the slit channels. Then just central part of the beam cross sections with sizes $0.4 \times 7 \mathrm{~cm}$ having minimal pitch angles of the electrons, enters the channels (see Fig. 5). The sheet beam thickness was $0.4 \mathrm{~cm}$ and the distance between the channel walls was $0.9 \mathrm{~cm}$.

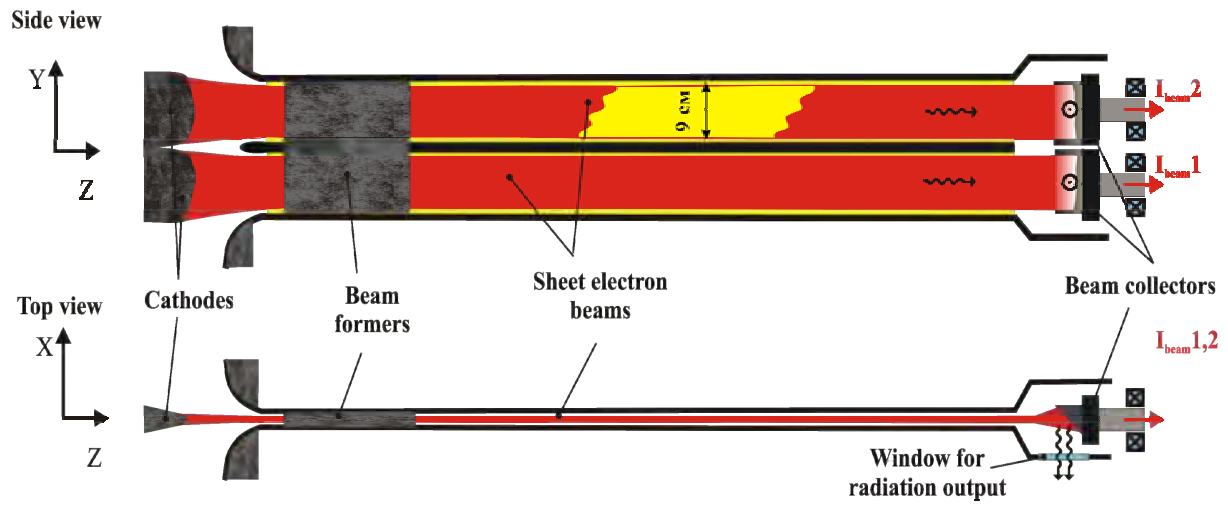

Fig. 5. Schematic drawing of the experiments on simultaneous generation of two sheet beams

Gap between the beam bounds and the channel walls should provide possibility of the beam oscillations under the transverse undulator field without contact of electrons with the channel walls. After transport through the $140 \mathrm{~cm}$ long channels with the magnetic field 
1.7 $\mathrm{T}$ the beams are dumped in the graphite collectors placed in the decreased magnetic field in the described experiments.

Typical traces of the diode voltage and the beam currents measured on the collectors are presented in Fig. 6. It is clearly seen that the time dependences of the beams currents are practically the same but the values have some difference. To understand this difference and to discover possible deformation of the beams cross sections the registration of the beam cross section profile on a thin ( $1 \mathrm{~mm}$ thickness) stainless steel plates have been used. These plates were mounted on special holders inside the channels. Due to the beam exposure the material of the plate heated up to evaporation creating the trace close to the beam cross section. Really this trace was slightly large than the beam size due to the trace edges melting. The reason of the beams currents difference in the shot presented in Fig. 6, was explored by analysis of the beams traces. As a result it was discovered that this difference was caused by tilt of the guiding magnetic field lines about the direction of the channel axis at the angle $\sim 0.01 \mathrm{rad}$.

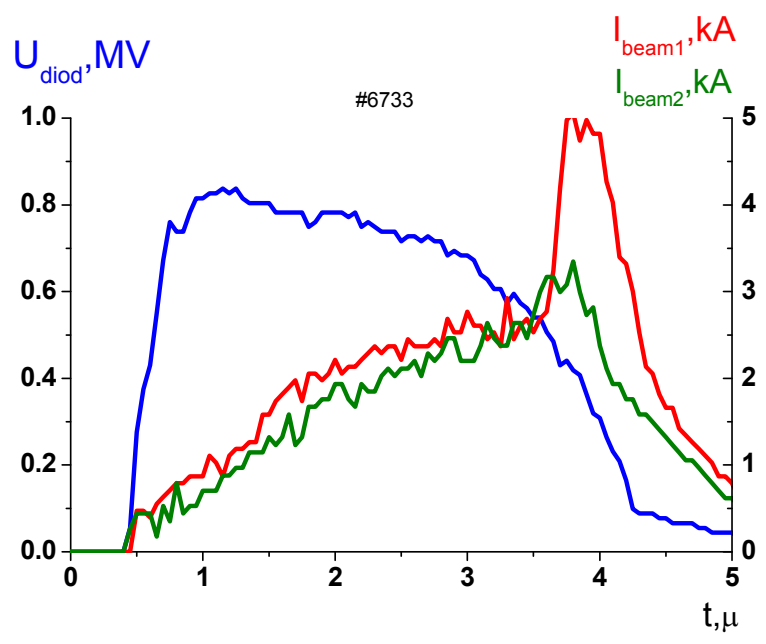

Fig. 6. Traces of the diode voltage and currents of two beams at the exit of the channels.

To eliminate this defect in the magnetic field geometry, concerned with inaccuracy in winding of magnetic coil, the special correcting coil was installed. This coil eliminated the tilt of the magnetic field without any damage in the beam cross section shape.

After that good coincidence of the beams currents has been achieved. Taking into account the results of computer simulations the analysis of drift displacements of the ends of the beams cross sections and their shape deformations (see imprints of the beam in the Fig.7) has shown that the beam space charge neutralization $f$ is larger than $1 / \gamma^{2}$ but far from unity. Since the initial thickness of the sheet beams was not equal to equilibrium quantity, some deformations of the beam cross sections at the transport length $140 \mathrm{~cm}$ have been observed in accordance with the simulation results. At the same time for the transport length $50 \mathrm{~cm}$ the ribbon shape of the beam cross section was good enough, and the gap 
between the beam border and the channel walls was still about $0.1 \mathrm{~cm}$. Thus any deviations of the beam cross section shape along $70 \mathrm{~cm}$ channel section after the beam former, where FEM resonator will be placed, seems to be negligible.

\section{Beam imprints on metal foils (at the channel exit) and graphite rods (in the channel center).}

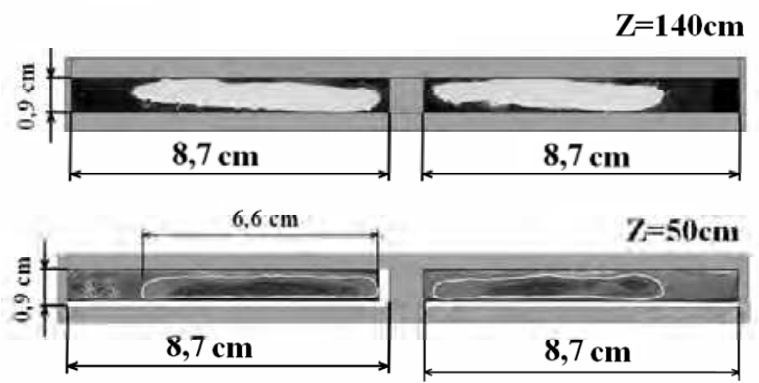

The beam shape remains acceptable for sub-mm generation on the length $50-70 \mathrm{~cm}$

Fig. 7. Cross section shape of two beams for two positions with a different distance $Z$ from the accelerator diode. Low pictures demonstrate the imprints of the two beams on graphite plates mounted in the channels at the distance $Z=50 \mathrm{~cm}$. Up pictures demonstrate the imprints on titanium foils at the exit of the channels with the distance $Z=140 \mathrm{~cm}$.

\subsection{Prospects of the proposed experiments at ELMI-device}

For the two-stage process of generation of submillimeter radiation we plan to use the ELMIdevice where the planar free electron maser generates coherent 4-mm radiation with appropriate power (Arzhannikov et al., 2008). Typical oscillograms of voltage pulses at the accelerating diode, the electron beam current, and the signal from the detector of 4-mm radiation are shown in Fig. 8a for the experiments described in (Arzhannikov et al., 2008). The significant level of the microwave signal at the detector was observed when the beam current exceeded $1 \mathrm{kA}$, in good agreement with the calculated starting current of the generator. The use of the scatterers of transverse wave beams in the two-dimensional Bragg mirror made it possible, for a large number of pulses, to obtain a narrowband generation at a frequency close to the frequency of the mode of the hybrid Bragg resonator. For example, the heterodyne analysis in Figs. $8 \mathrm{~b}$ and $8 \mathrm{c}$ shows that the radiation spectrum is localized near a frequency of $75.3 \mathrm{GHz}$ during almost entire 300-ns duration of the pulse. This corresponds to the excitation of the single-resonator mode. A number of other shots showed the generation at frequencies of 74.9, 75.1, and 75.5 GHz corresponding to the excitation of other longitudinal modes of the resonator. Moreover, the simultaneous excitation of a few longitudinal modes was also observed. According to the simulation results, the possibility of exciting different modes is due to variations in the electron energies and beam current over the pulse, as well as pulse-to-pulse variations in the beam parameters. The analysis of the 
time behaviour of the plasma fluorescence in the channel indicates that the total duration of the microwave signal is limited due to the arrival of the collector plasma at the radiation deflector.
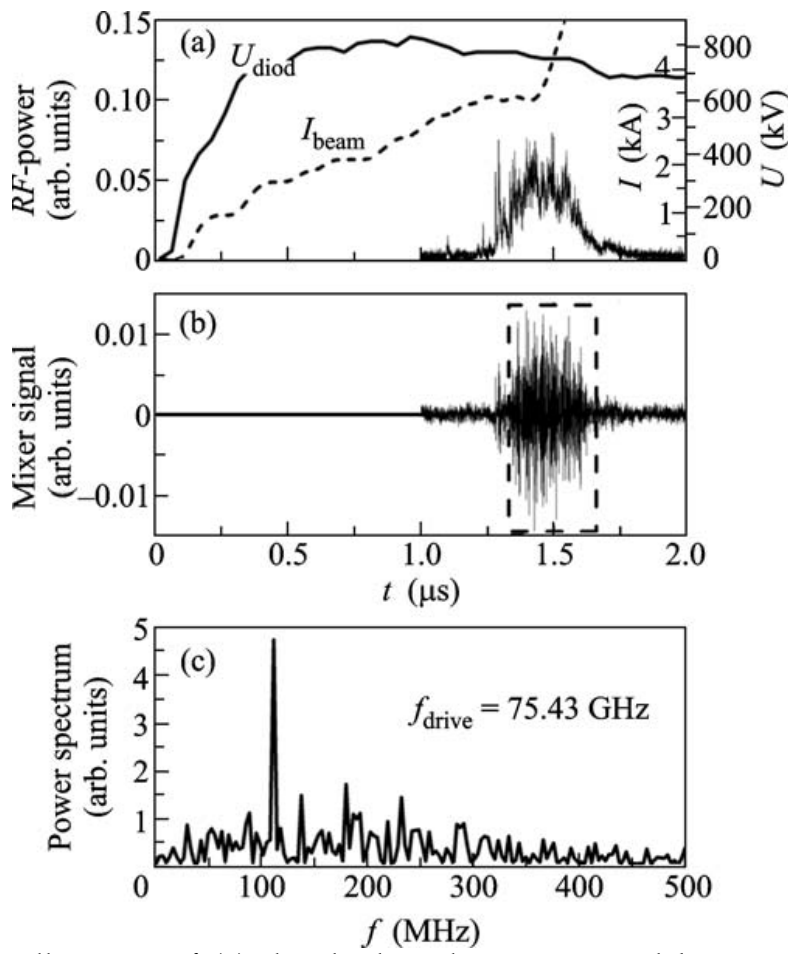

Fig. 8. Typical oscillograms of (a) the diode voltage $U_{\text {diod }}$ and beam current $\mathrm{I}_{\text {beam }}$ in the microwave pulse, (b) the mixer signal, and (c) the radiation spectrum.

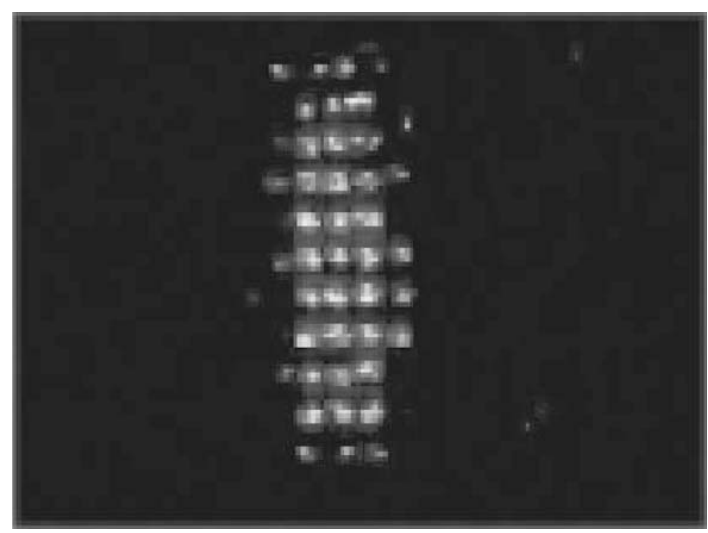

Fig. 9. Photograph of the fluorescence of the neon-tube panel exposed to the microwave pulse. 
The total power of the output radiation of about a few tens of megawatts was estimated using the readings of the calorimeter and the signals from the calibrated hot-carrier detectors with allowance for the angular pattern of the output radiation determined by the fluorescence pattern of a neon-tube panel placed at various distances from the output window of the generator (see Fig. 9). The field structure similar to the $H 1,0$ wave confirms the theoretical conclusion on the uniform distribution of the comoving-wave field over the cross section at the output from the interaction space.
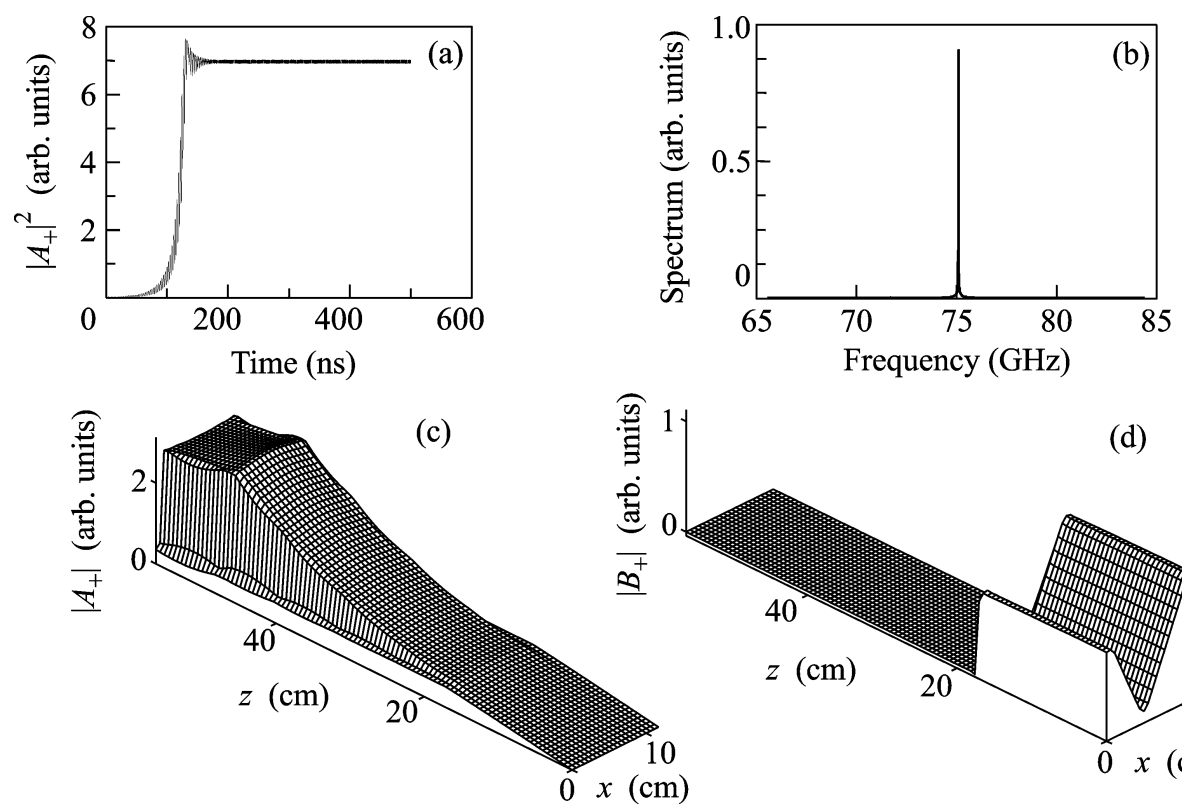

(d)

Fig. 10. Simulation of the radiation synchronization in a planar free-electron maser with the combined resonator comprising one and two-dimensional Bragg mirrors: (a) the time dependence of the normalized output power $|A+| 2$ under the conditions of a singlemode, single-frequency generation, (b) output radiation spectrum, and the steady-state spatial structure of the partial-wave fields (c) $|A+|$ and (d) $|B+|$.

Fig. 10 shows the results of simulating the generation and spatial synchronization of radiation for the electrodynamic-system configuration and the electronbeam parameters close to the respective experimental conditions. The most important key result is the conclusion that a given spatial distribution of the fields, which is determined only by the system parameters and is independent of the initial conditions, is established during the development of self-excited oscillations at an arbitrary initial noise modulation of the electron beam or small initial perturbations of the electromagnetic fields. In this case, the output-radiation front associated with the wave $A+$ has a deterministic (i.e., not random) phase distribution over the transverse coordinate $x$. It is also important to emphasize that the partial wave $A+$ synchronous to the electron beam in the steady generation regime has an almost uniform field distribution over the $x$ coordinate (see Fig. 10c). This ensures the same conditions for the energy extraction from all of the beam components. Variation in the 
electron energy, i.e., in the parameter $\square$, results in a stepwise change in the frequency of the generated radiation, which corresponds to the excitation of modes with various numbers of field variations over the longitudinal coordinate $z$.

Now we are starting the experimental realization of the variant of the two-stage submillimeter generation presented in Fig. 2 for the case that does not include any mirrors for submm radiation. In this case we can measure a level of super-radiation in the terahertz band from the channel \#2 while 4-mm radiation is generated in the channel \#1 by the free electron maser mechanism. It was cleared up by computer simulations and experimental studies that the conditions of the stationary state of the 4-mm generation in the ELMIexperiments can be achieved in time $100 \div 200 \mathrm{~ns}$ and the frequency of generated radiation should be unchanged even when E-beam energy is varied in $50 \mathrm{keV}$. It means that we may realize a two-stage THz-generation process at the ELMI-device as soon as E-beam parameters are approximately not changed in time $200 \div 300 \mathrm{~ns}$.

The important feature of our experiments is to use high density of 4-mm waves accumulated in the resonator of the FEM-oscillator (channel \#1 in Fig.2) as a pumping force in the electrodynamics undulator (channel \#2 in Fig.2) of the FEL-generator. For the pulse duration about of $0.5 \mu$ s the power flow density of the 4-mm radiation in the FEM-oscillator can be achieved $I_{0}=0.1 \div 0.5 \mathrm{GW} / \mathrm{cm}^{2}$ and the same level of the $4-\mathrm{mm}$ radiation will be in the FEL-generator.

When the wavelength of the pumping FEM radiation is $\lambda_{0}=4 \mathrm{~mm}$ and the E-beam relativistic factor is $\gamma=3$ so that the Doppler parameter of wavelength shortening is $\lambda \approx \lambda_{0} /\left(4 \gamma^{2}\right)$ then the spatial growth rate for the second stage is estimated as (Arzhannikov et al., 2006)

$$
G\left[\mathrm{~cm}^{-1}\right] \approx 7\left(\frac{j_{b}[\mathrm{kA} / \mathrm{cm}]}{b[\mathrm{~mm}] \lambda_{0}[\mathrm{~mm}]}\right)^{1 / 3} \cdot \frac{a_{0}^{2 / 3}}{\gamma},
$$

where $\mathrm{jb}$ is the current density per unit transverse size of the sheet beam (linear current density), $\mathrm{b}$ is the gap between the vacuum channel plates, and

$$
a_{0}=2.3 \cdot 10^{-8} \lambda_{0}[\mathrm{~mm}] \cdot E[\mathrm{~V} / \mathrm{cm}]
$$

is the pump wave parameter, $\mathrm{E}$ is the pump $4-\mathrm{mm}$ wave amplitude. For our case this amplitude is estimated on the level $\mathrm{E}=1 \mathrm{MV} / \mathrm{cm}$ and the pump wave parameter $a_{0}=5 \cdot 10^{-2}$ $10^{-1}$.

Thus, for the relativistic factor $\gamma=3$, the linear current density $j_{b}=1 \mathrm{kA} / \mathrm{cm}$ and the gap $\mathrm{b}=10 \mathrm{~mm}$ the spatial rate of sub-mm radiation is estimated as $\mathrm{G} \approx 0.1 \mathrm{~cm}^{-1}$.

Let us look at the experimental limitations due to beam quality. A "cold" beam is defined by the following expression $S<<1$, where $S=\delta V_{z} /\left(V_{z 0}-V_{\text {wave }}\right), \delta V_{z}$ is the beam electron velocity spread, $V_{z 0}-V_{\text {wave }}$ is difference between the beam velocity and the wave one. If $S>>1$ then terahertz radiation is generated incoherently. For our case the requirement $S<<1$ can be expressed in the form:

$$
\frac{\delta V_{z}}{c}<<\frac{\lambda_{0} \cdot G}{4 \cdot \gamma^{2}}=2 \cdot 10^{-5} .
$$

Such a small value of the E-beam longitudinal velocity spread can be reached by a very accurate choice of geometry of magnetic field configuration in accelerator diodes and an appropriate strength of the guiding magnetic field (Arzhannikov et al., 2006). 


\section{Conclusion}

Thus, theoretical and experimental studies demonstrate the operability of the twodimensional distributed feedback and the possibility of use this spatial synchronization mechanism to generate the high-power mm-wave narrowband radiation.

It is important to note that the two-dimensional distributed feedback can be used for the spatial synchronization of radiation generated by several electron beams in multichannel planar FEM devices to produce microwave radiation of GW power level.

High level of the power density with its homogeneous distribution in very large volume for the case of the planar FEM with the two-dimensional distributed feedback allows one to use this electrodynamics system to generate THZ-band radiation on the base intercavity stimulated scattering of mm-wave.

Experiments carried out at the ELMI-device have shown that the sheet beams of 1-Mev electrons are produced with parameters appropriate not only for the generation of highpower 4-mm wave but also for production of submillimeter radiation through backscattering process.

\section{References}

Arzhannikov A.V., V.T.Astrelin, V.A.Kapitonov, M.P.Lyamzin, S.L.Sinitsky, M.V.Yushkov (1990) Sudies of microsecond ribbon REB generation and transport, Proceedings of the 9 International Conference on High-Power Particle Beams, Novosibirsk, USSR, 1990, Vol.1, pp.256-263

Arzhannikov A.V., S.L.Sinitsky (1996) Reduction of angular spread at nonadiabatic electron motion in magnetically insulated diode. Proceedings of the 11-th International Conference on High Power Particle Beams, Prague, Chech Republic, 1996. Vol.1, pp.367-370

Arzhannikov A.V., Ginzburg N.S., Nikolaev V.S., Peskov N.Yu., Sergeev A.S., Sinitsky S.L.,.Zotkin R.P (1992) FEL driven by high current ribbon REB and operated with two dimensional feedback, Technical Digest of the 14th Intern. FEL Conference, p.214, August 1992, Kobe, Japan.

Arzhannikov A.V., Ginzburg N.S., Peskov N.Yu., ., Sergeev A.S, Sinitsky S.L. (1995).Superpower free-electron lasers with two-dimension distributed feedback,. Nuclear Instruments and Methods in Physics Research, v.A358, pp.189-192, 1995.

Arzhannikov A.V.; Bobylev V.B.; Ginzburg N.S.; Ivanenko V.G.; Kalinin P.V.; Kuznetsov S.A.; Peskov N.Y.; Sergeev A.S.; Sinitsky S.L.; Stepanov V.D. (2003), Single-Channel and Multi-Channel Planar Free-Electron Masers, Radiophysics and Quantum Electronics, 46 (2003), 10, pp. 810-815.

Arzhannikov A.V., Ginzburg N.S., Kalinin P.V., Kuznetsov S.A., Peskov N.Yu., Rozental R.M., Sergeev A. S., Sinitsky S.L., Stepanov V.D., Thumm M., Zaslavsky V. Yu., Zotova I.V.. Intercavity Scattering Scheme for Two-stage Generation of Submillimeter Radiation on the Base of Planar 2D Bragg FEM (2006), Proceeding of International Workshop "Strong Microwaves in Plasmas", Nizhny Novgorod, Russia, 25 July- 1 August 2006, v. 1, pp. 228-232. 
Arzhannikov A.V., Astrelin V.T., Kalinin P.V., Sinitsky S.L., Stepanov V.D. (2007) Simultaneous generation and transport of two high-current sheet beams. Vestnik NGU, Seria: Fizika, (Novosibirsk State University bulletin, Serial: Physics),Vol. 2, No 4, 2007, pp.125-131 (in Russian).

Arzhannikov A. V., Astrelin V. T., Ginzburg N. S., Kalinin P. V., Kuznetsov A. S., Kuznetsov S. A., Peskov N. Yu., Sergeev A. S., Sinitsky S. L., Stepanov V. D., Zaslavsky V. Yu., Zotova I. V. (2007) Submillimeter radiation production by intercavity stimulated scattering in planar FEM at the ELMI-device, Conference Digest of the Joint 32nd International Conference on Infrared and Millimetre Waves, and $15^{\text {th }}$ International Conference on Terahertz Electronics «IRMMW-THz 2007», Cardiff, UK, 3rd - 7th September, 2007, Vol. 2, pp. 835-836.

Arzhannikov A.V., Ginzburg N.S., Zaslavsky V.Yu., Ivanenko V.G., Ivanov I.A., Kalinin P.V., Kuznetsov A.S., Kuznetsov S.A., Peskov N.Yu., Sergeev A.S., Sinitsky S.L., Stepanov V.D. (2008), Generation of Spatially Coherent Radiation in Free-Electron Masers with Two-Dimensional Distributed Feedback, ISSN 0021-3640, JETP Letters, 2008, Vol. 87, No. 11, pp. 618-622. (C Pleiades Publishing, Ltd., 2008.

Ginzburg N.S., Peskov N.Yu., Sergeev A.S., Phelps A.D.R., Konoplev I.V., Robb G.R.M., Cross A.W. Arzhannikov A.V., Sinitsky S.L. Theory and design of a free-electron maser with two-dimensional feedback driven by a sheet electron beam. Physical Review E (Statistical Physics, Plasmas, Fluids, and Related Interdisciplinary Topics), Volume 60, Issue 1, July 1999, pp.935-945

N.S.Ginzburg, N.Yu.Peskov, A.S.Sergeev, A.V.Arzhannikov, S.L. Sinitsky (2001) Novel scheme of multi-beam FEL synchronized by two-dimensional distributed feedback, Nuclear Instruments and Methods in Physics Research Section A: Accelerators, Spectrometers, Detectors and Associated Equipment, v. A475 pp.173-177, December 2001.

Dobroiu A., Yamashita M., Ohshima Y.N., Morita Y., Otani C., Kawase K. (2004) The backward wave oscillator as a radiation source in terahertz imaging, Conference Digest of the 2004 Joint 29th International Conference on Infrared and Millimeter Waves, 2004 and 12th International Conference on Terahertz Electronics, pp. 825 - 826, Karlsruhe, Germany, University of Karlsruhe (TH), Oct. 2004. ISBN-0-7803-8490-3.

Kohler R., Tredicucci A., Beltram F., Beere H., Linfield E., Davies G., Ritchie D., Iotti R., Rossi F. (2002) Terahertz semiconducting-heterostructure laser, Nature 417, 156-159 (9 May 2002), Macmillan Magazines Ltd.

Minehara E., Hajima R., Iijima H., Kikuzawa N., Nagai R., Nishimori N., Nishitani T., Sawamura M., Yamauchi T., (2005). Jaery 10KW High Power ERL-FEL and Its Applications in Nuclear Energy Industries, Proceedings of the 27th International Free Electron Laser Conference, , pp. 305 - 308, 21-26 August 2005, Stanford, California, USA.

Vinokurov N.A., Gavrilov N.G., Knyazev B.A., Kolobanov E.I., Kotenkov V.V., Kubarev V.V., Kulipanov G.N., Matveenko A.N., Medvedev L.E., Miginsky S.V., Mironenko L.A., Oreshkov A.D., Ovchar V.K., Popik V.M., Salikova T.V., Scheglov M.A., Serednyakov S.S., Shevchenko O.A., Skrinsky A.N., Tcheskidov V.G. (2006), Status of the Novosibirsk High Power Terahertz FEL, Proceedings of the 28th International Free Electron Laser Conference FEL 2006, pp. 492 - 495, August 27 - September 1, 2006, BESSY, Berlin, Germany. 
Sinitsky S.L., Arzhannikov A.V., Astrelin V.T., Kalinin P.V., Stepanov V.D. (2008) Simultaneous Generation and Transport of Two Microsecond Sheet REBs in Application to Multichannel FEM. Proceedings of the 17th International Conference on High-Power Particle Beams, Mianyang, Sichuan, P.R. China, July 6-11, 2008, pp. 235238. 


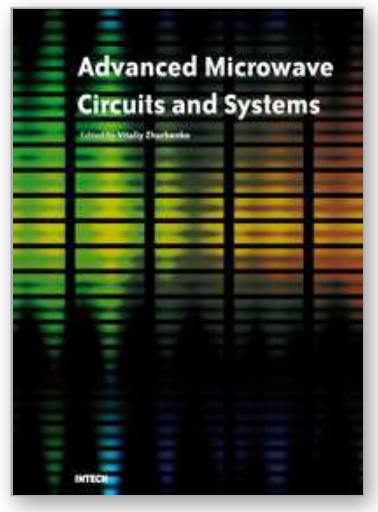

\author{
Advanced Microwave Circuits and Systems \\ Edited by Vitaliy Zhurbenko
}

ISBN 978-953-307-087-2

Hard cover, 490 pages

Publisher InTech

Published online 01, April, 2010

Published in print edition April, 2010

This book is based on recent research work conducted by the authors dealing with the design and development of active and passive microwave components, integrated circuits and systems. It is divided into seven parts. In the first part comprising the first two chapters, alternative concepts and equations for multiport network analysis and characterization are provided. A thru-only de-embedding technique for accurate onwafer characterization is introduced. The second part of the book corresponds to the analysis and design of ultra-wideband low- noise amplifiers (LNA).

\title{
How to reference
}

In order to correctly reference this scholarly work, feel free to copy and paste the following:

Andrey Arzhanikov (2010). Intercavity Stimulated Scattering in Planar FEM as a Base for Two-Stage Generation of Submillimeter Radiation, Advanced Microwave Circuits and Systems, Vitaliy Zhurbenko (Ed.), ISBN: 978-953-307-087-2, InTech, Available from: http://www.intechopen.com/books/advanced-microwavecircuits-and-systems/intercavity-stimulated-scattering-in-planar-fem-as-a-base-for-two-stage-generation-ofsubmillimeter-

\section{INTECH}

open science | open minds

\section{InTech Europe}

University Campus STeP Ri

Slavka Krautzeka 83/A

51000 Rijeka, Croatia

Phone: +385 (51) 770447

Fax: +385 (51) 686166

www.intechopen.com

\section{InTech China}

Unit 405, Office Block, Hotel Equatorial Shanghai

No.65, Yan An Road (West), Shanghai, 200040, China

中国上海市延安西路65号上海国际贵都大饭店办公楼 405 单元

Phone: +86-21-62489820

Fax: +86-21-62489821 
(C) 2010 The Author(s). Licensee IntechOpen. This chapter is distributed under the terms of the Creative Commons Attribution-NonCommercialShareAlike-3.0 License, which permits use, distribution and reproduction for non-commercial purposes, provided the original is properly cited and derivative works building on this content are distributed under the same license. 